\title{
The Development of Hypertension in Rats with Intraventricular Grafts of Fetal SHR or WKY Hypothalamus
}

\author{
C.A. Murphy", R. Canbeyli and B.G. Yongue \\ Department of Developmental Psychobiology, Columbia University, 722 W. 168th Street, New York, NY \\ 10032 and "Department of Anatomy and Neurobiology, Medical College of Pennsylvania, \\ 3200 Henry Avenue, Philadelphia, PA 19129, USA
}

To evaluate the role of the hypothalamus in
hypertension, we compared the development of
lood pressure (BP) in normotensive host rats
grafted at weaning with fetal hypothalamus from
genetically hypertensive or normotensive
donors. The anteroventral region of the third
ventricle (AV3V) has been implicated in the
control of BP, such that lesions of AV3V pre-
vent or attenuate hypertension in a number of
animal models. Recent studies have shown that
BP is elevated after grafting SHR hypothalamus
into the AV3V region of genetically normo-
ensive hosts. WKY hosts grafted with fetal SHR
hypothalamus (E15) exhibited increased BP for
over 5 months after implantation, while hosts
eceiving WKY hypothalamus experienced only
transient rise in BP at 1 month post-implant 1/. Normal Wistar hosts grafted with older (E19-20) SHR hypothalamus exhibited increased BP at 1 month post-implant, and not at subsequent ages, while E19-20 WKY hypothalamus did not influence BP at any age $/ 2 /$. The following experiments were designed to determine how the pressor effects of grafting SHR hypothalamus may vary as a function of the embryonic age and specific anatomical origin of grafted cells.

Two normotensive rat strains were used as hosts: outbred Wistar and inbred Wistar-Kyoto (WKY) rats. At 25 days of age, hosts were grafted in the AV3V region with E15-16 fetal SHR hypothalamus, SHR cortex or WKY hypothalamus. Sham-operated controls received infusions of $0.9 \%$ saline. Systolic blood pressure was measured at 30,60, 90 and 120 days of age. Based on evidence that the AV3V region also mediates behavioral control of fluid/electrolyte homeostasis, daily consumption of water and $1.5 \%$ saline was also measured at 60 and at 120 days of age.

In Wistar hosts (Fig. 1), all grafts, regardless of strain or anatomical origin, increased blood pressure at 60 days. Hypertension was sustained at 90 and 120 days in Wistar rats receiving SHR grafts of either type, but not in hosts grafted with WKY hypothalamus. Similarly, WKY hosts grafted with either SHR hypothalamus or SHR cortex had higher BP $(158 \mathrm{mmHg})$ by 90 days than either sham control $(140 \mathrm{mmHg})$ or hosts receiving WKY hypothalamus $(120 \mathrm{mmHg})$. On the whole, WKY graft recipients exhibited more modest and less permanent increases in BP than Wistar hosts, perhaps due to specific immunological incompatibilities between the two inbred strains.

Both hypothalamus and cortex obtained from E15-16 SHR fetuses elicited chronic elevations in BP. In contrast, earlier work showed that grafts of E19-20 SHR hypothalamus increased BP at 60 days of age while SHR cortex did not. Differences in the anatomical specificity of the transplant preparation at different embryonic ages suggests that E15-16 cortical cells may be sufficiently undifferentiated that when grafted into the AV3V, they are induced to function as hypothalamic cells.

Wistar hosts grafted with E15-16 SHR hypothalamus or SHR cortex also consumed significantly less water $(p<0.01)$ at 60 days (SHR cortex: $19 \mathrm{ml}$; SHR hypothalamus: $18 \mathrm{ml}$ ) than rats grafted with either WKY hypothalamus (27 $\mathrm{ml})$ or saline $(34 \mathrm{ml})$. These data suggest a potentially informative association between graft-induced alterations in fluid intake at 60 days and subsequent chronic elevations in BP. At 60 days, even hosts grafted with WKY hypothalamus had high BPs; however, only recipients of SHR grafts consumed less water at 
this age. Decreased water intake may reflect some aspect of a compensatory response to developing hypertension (e.g. a response to increased blood volume).

The fact that both SHR and WKY grafts elicit transient increases in BP suggests multiple actions of hypothalamic cells, some strainspecific and some not, in influencing BP. Potential mechanisms resulting in the pressor and behavioral effects of grafting SHR hypothalamus may reflect strain differences in specific hypothalamic neuroendocrine and neurochemical factors (e.g. angiotensin II, vasopressin, atrial natriuretic peptide). With further investigation, the SHR-transplant preparation should develop into a useful experimental model of neurallymediated hypertension.

\section{REFERENCES}

1. Eilam R, Malach R, Bergmann F, Segal M. Hypertension induced by hypothalamic transplantation from genetically hypertensive to normotensive rats. J Neurosci 1991; 11: 401-411.

2. Murphy C, Yongue B, Cheng J, Skaredoff L. Cardiovascular and behavioral effects of fetal hypothalamic grafts in hypertensive and normotensive rats. Abstract presented at the Society for Neuroscience meeting, 1991.

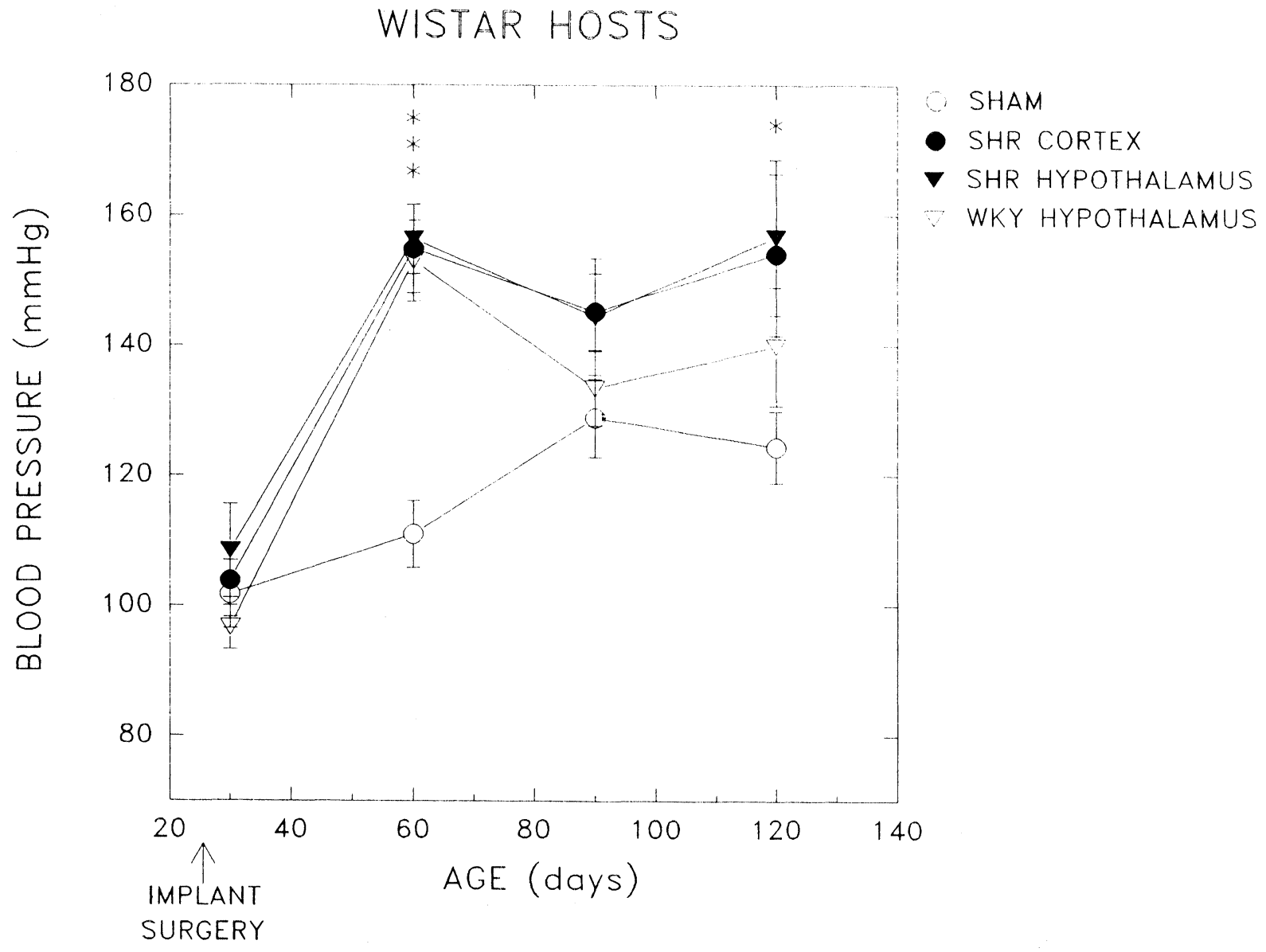

Fig. 1: Systolic blood pressures of Wistar hosts receiving sham infusions of $0.9 \%$ saline, grafts of SHR cortex, SHR hypothalamus or WKY hypothalamus at 25 days of age.

* SHR grafts vs. sham group, $p<0.02$

*** All grafts vs. sham group, $p<0.001$ 

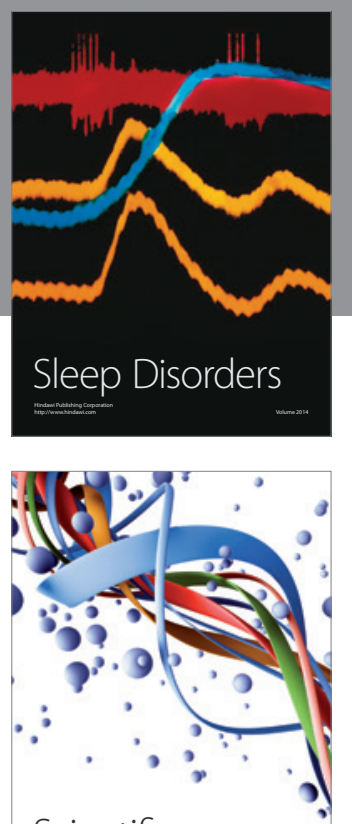

Scientifica
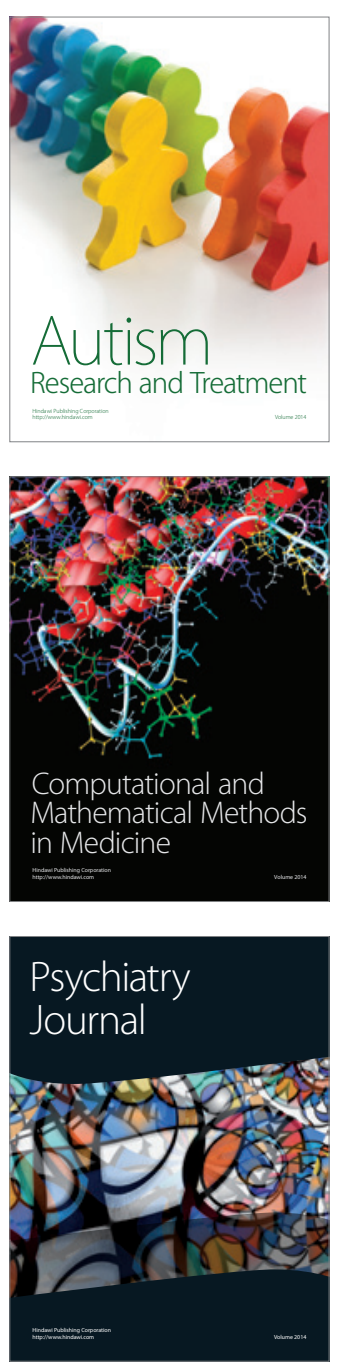
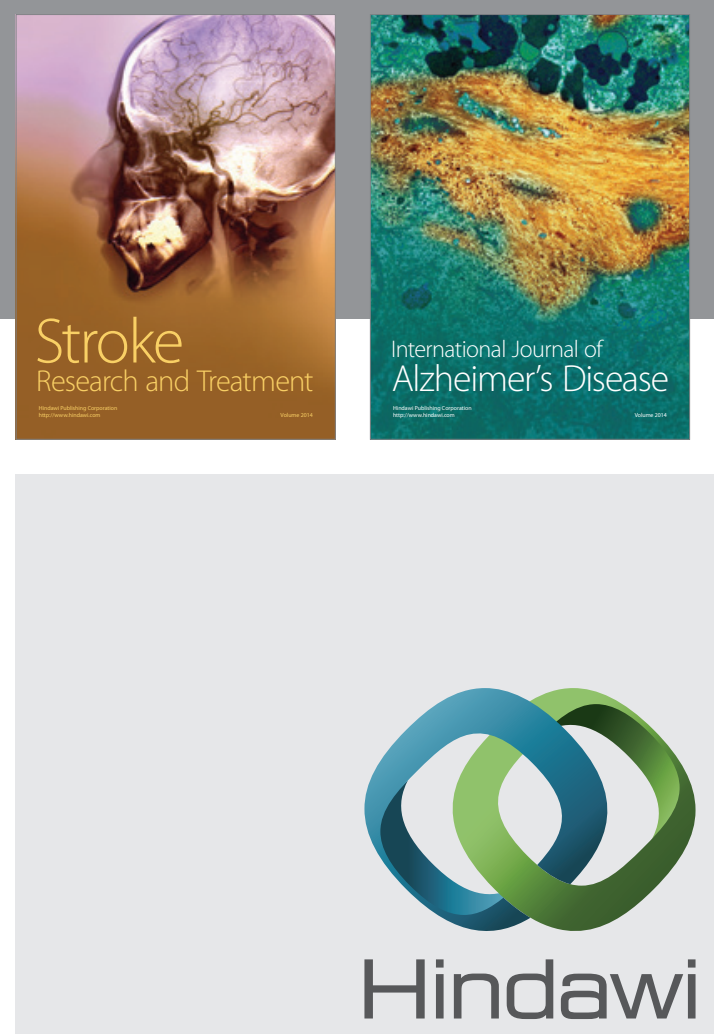

Submit your manuscripts at

http://www.hindawi.com
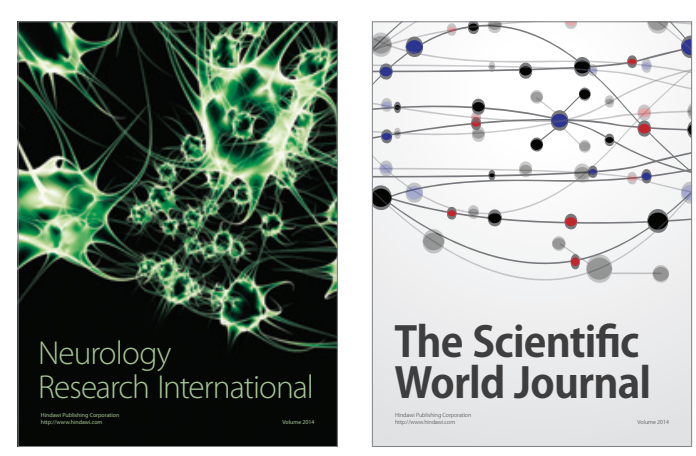

The Scientific World Journal

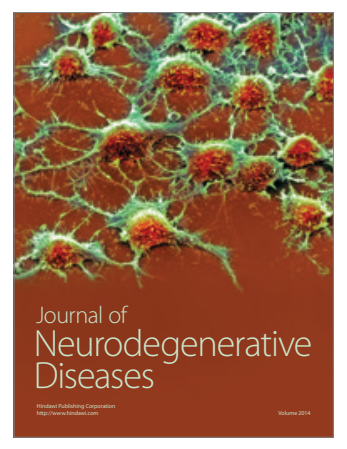

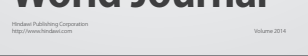

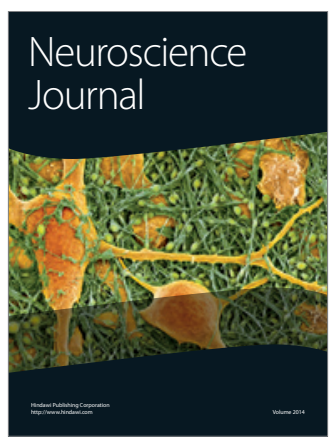

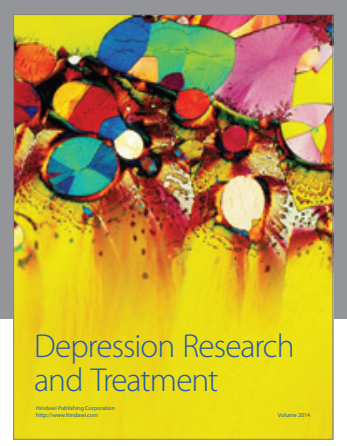
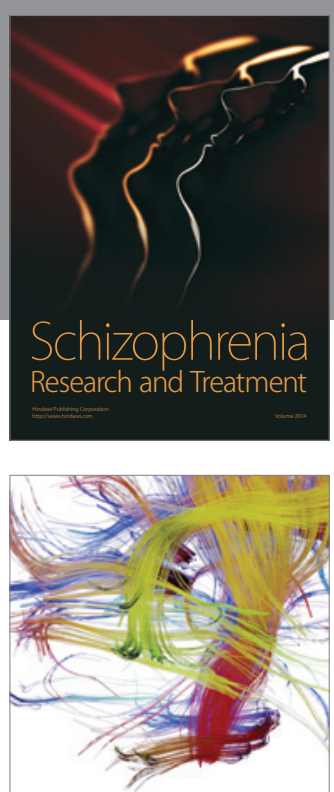

Brain Science

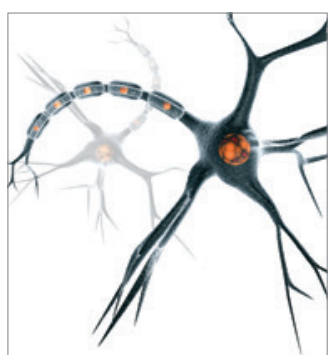

Neural Plasticity
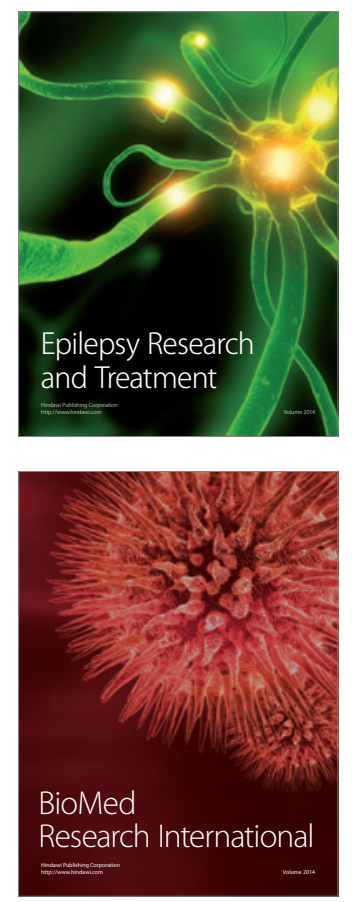

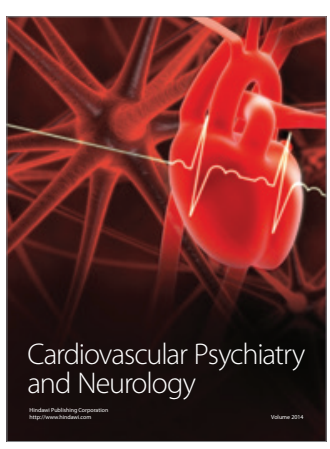

Parkinson's

Disease
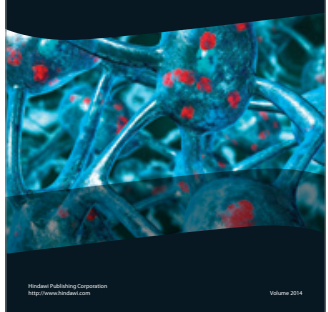